\title{
Avaliação de resultados da psicoterapia psicanalítica
}

\author{
Assessment of psychoanalytic psychotherapy outcomes
}

\author{
Simone Isabel Jung¹, Maria Lúcia Tiellet Nunes², Cláudio Laks Eizirik ${ }^{3}$ \\ ${ }^{1}$ Psicóloga. Mestre em Ciências Médicas-Psiquiatria, Universidade Federal do Rio Grande do Sul (UFRGS), Porto Alegre, RS. ${ }^{2}$ Psicóloga. Doutora \\ em Psicologia, Universidade Livre de Berlim, Berlim, Alemanha. Professora titular, Faculdade de Psicologia, Pontifícia Universidade Católica do \\ Rio Grande do Sul (PUCRS), Porto Alegre, RS. Coordenadora, Programa de Pós-Graduação em Psicologia, Faculdade de Psicologia, PUCRS, Porto \\ Alegtre, RS. ${ }^{3}$ Médico psiquiatra. Doutor em Medicina, UFRGS, Porto Alegre, RS. Professor adjunto, Departamento de Psiquiatria e Medicina Legal, \\ UFRGS, Porto Alegre, RS. Analista didata, Sociedade Psicanalítica de Porto Alegre, Porto Alegre, RS. Presidente, International Psychoanalytic \\ Association (IPA) \\ Simone Isabel Jung recebeu bolsa CAPES de 03/2005 a 02/2006.
}

\section{Resumo}

Introdução: Este artigo relata uma investigação de coorte retrospectiva que avaliou a efetividade da psicoterapia psicanalítica com pacientes adultos em um serviço de atendimento comunitário na cidade de Porto Alegre, Brasil.

Método: A amostra foi constituída por 34 pacientes divididos em dois grupos: grupo 1, 17 pacientes que realizaram tratamento por até 11 meses (média de 6,4 meses), e grupo 2, 17 pacientes com 1 ano ou mais de psicoterapia (média de 24,7 meses), contatados após o término de seu tratamento psicoterápico, em média 20,9 meses no grupo 1 e 29,9 meses no grupo 2. Os instrumentos utilizados foram: entrevista semi-estruturada, questionário de efetividade e escala de avaliação global do funcionamento. Experts independentes aplicaram a escala de avaliação global do funcionamento na entrevista inicial de tratamento (realizada pelo psicoterapeuta e encontrada no arquivo da instituição) e de seguimento.

Resultados: Os pacientes melhoraram significativamente seu funcionamento global, comparando a avaliação inicial à de seguimento da psicoterapia $(\mathrm{p}<0,001)$, independentemente do grupo de tratamento. Pacientes e experts avaliaram o tratamento psicoterápico de forma satisfatória. Entretanto, a opinião dos experts não apresentou correlação com a opinião dos pacientes investigados.

Conclusões: A psicoterapia psicanalítica foi efetiva na amostra estudada. A duração da psicoterapia, analisada isoladamente, não foi um fator decisivo para o resultado do tratamento, isto é, outros fatores também devem ser considerados na avaliação de resultados (relação terapêutica, qualidade das relações objetais, potencial auto-reflexivo, reação às interpretações). Constatou-se que a maioria dos pacientes fica satisfeita com resultados em um tempo menor do que aquele que seus psicoterapeutas considerariam ideal.

Descritores: Psicoterapia, terapia psicanalítica, avaliação de resultados (cuidados de saúde), avaliação de resultado de intervenções terapêuticas, pesquisa.

\begin{abstract}
Introduction: This article reports a retrospective cohort investigation, which assessed the effectiveness of psychoanalytic psychotherapy in adult patients at a community health center in the city of Porto Alegre, Brazil.

Methods: The sample was composed of 34 patients, who were divided into two groups: group 1, 17 patients who underwent treatment for up to 11 months (mean 6.4 months) and group 2, 17 patients who underwent psychotherapy for 1 year or longer (mean 24.7 months). The patients were contacted after their psychotherapeutic treatment was over, in average 20.9 months (group 1) and 29.9 months (group 2). Instruments used were semi-structured interview, effectiveness questionnaire and global assessment of functioning scale. Independent experts applied the global assessment of functioning scale in the initial interview (carried out by the psychotherapist and found in the institution's files), as well as in the follow-up interview.

Results: The patients significantly improved their global functioning, when initial assessment was compared to psychotherapy follow-up ( $<<0.001$ ), regardless of treatment group. Patients and experts considered psychotherapy satisfactory. However, the experts' opinion was not related to the patients' opinion.
\end{abstract}


Conclusions: Psychoanalytic psychotherapy was effective in the studied sample. Length of psychotherapy, when assessed alone, was not a decisive factor for treatment outcome, i.e., other factors should also be taken into account when assessing outcomes, such as therapeutic relationship, quality of object relations, self-awareness potential and reaction to interpretations. Most patients were found to be satisfied with outcomes in a shorter time than their psychotherapists would consider optimal.

Keywords: Psychotherapy, psychoanalytic therapy, outcome assessment (health care), outcome assessment of therapeutic interventions, research.

\section{Introdução}

Apesar do estado atual do conhecimento psicanalítico e da relevância dos estudos já realizados, as opiniões em relação à efetividade da psicoterapia psicanalítica (PP) ainda são divergentes, tanto na mídia em geral ${ }^{1-3}$ quanto entre os profissionais da chamada área "psi" "-7.7 Nesse contexto, a investigação sistemática dos resultados em PP ainda é uma necessidade ${ }^{6}$, principalmente como resposta às críticas referentes à duração e à qualidade dos resultados psicoterápicos ${ }^{5,7}$.

A avaliação da efetividade das psicoterapias teve seu início com o próprio Freud ${ }^{8}$, através do estudo de caso único, no seu trabalho Estudos sobre a histeria9. A partir desse estudo, um número significativo de informes de pesquisas de resultados em PP foram publicados, principalmente nos anos $2000^{10-19}$. Wallerstein ${ }^{20}$, por exemplo, já menciona quatro gerações de pesquisas psicanalíticas de resultados. Entretanto, em uma busca nas bases de dados LILACS, PubMed (período de janeiro de 1990 a abril de 2007) e PsycINFO (1989 a abril de 2007), encontram-se apenas quatro pesquisas de resultado com adultos (não-psicóticos e sem abuso de drogas) em PP ambulatorial de longa duração, desenvolvidas ou em desenvolvimento na América Latina $^{21-24} \mathrm{e}$, destas, uma no Brasil ${ }^{22}$. Portanto, as pesquisas de avaliação de resultados em psicoterapia no Brasil constituem um campo fértil para a investigação psicanalítica.

O estudo apresentado neste artigo é uma investigação de coorte retrospectiva aprovada pelo Comitê de Ética da Universidade Federal do Rio Grande do Sul (cadastro 20035) e faz parte da dissertação de mestrado da primeira autora.

O objetivo geral foi verificar a efetividade da PP em pacientes adultos que receberam tratamento em um serviço de atendimento comunitário. Foram analisadas as correlações entre a duração do tratamento e o resultado e entre a opinião do paciente e a de experts em PP.

É relevante mencionar que, cada vez mais, nas discussões sobre psicoterapia, os termos psicanalítica e psicodinâmica têm sido usados como sinônimos ${ }^{25}$. Portanto, neste estudo, os termos PP e psicoterapia psicodinâmica serão usados como equivalentes, designando a mesma modalidade de psicoterapia derivada da psicanálise.

\section{Método}

\section{Pacientes}

Os critérios para inclusão na pesquisa foram: idade mínima de 18 anos no início da psicoterapia; freqüência mínima de 18 sessões de tratamento; término de tratamento por alta (objetivos atingidos), interrupção (sem atingir todos os objetivos) e abandono (não comparecimento às sessões) ocorrido no período de 12/1999 a 07/2005 e, no mínimo, 6 meses antes da inclusão na pesquisa.

Foram excluídos pacientes com atividades profissionais ou de formação em psicologia e medicina, seguindo uma norma da instituição, e pacientes que retornaram para tratamento psicoterápico. Tais exclusões devem-se por questões éticas e para evitar possível fator de confusão na avaliação dos resultados.

A pesquisa foi planejada para incluir pacientes com, no mínimo, 28 sessões de tratamento, baseada nos estudos de dose/efeito de Howard et al. ${ }^{26}$ e Kopta et al. ${ }^{27}$, mas, no intuito de aumentar a amostra, foi reduzido o número de sessões. Através de um rastreamento do número de sessões utilizado em pesquisas de tratamentos psicodinâmicos de curta duração realizadas a partir do ano $2000^{28-39}$ e que apontam um amplo espectro de uma a 63 sessões, foi possível chegar ao número mínimo de 18 sessões, e cinco pacientes foram incluídos com essa freqüência.

O tempo de follow-up de 1 ano foi o planejado inicialmente, seguindo a tendência de outras investigações ${ }^{19}$. Entretanto, para tornar esta pesquisa factível no tempo disponível, incluíram-se casos com tempo menor de follow-up. Cinco pacientes foram incluídos com tempo de follow-up inferior a 1 ano.

Gabbard $^{25}$ propõe, como tratamentos de longa duração, aqueles com mais de 24 sessões ou mais que 6 meses de duração. Leichsenring ${ }^{40}$ refere que, na Alemanha, tratamentos entre 25 e 50 sessões não são considerados longos. Portanto, esforços para definir o que é um tratamento de longa duração podem ser $\operatorname{arbitrários}^{25,40}$. Consideraram-se de longa duração, neste estudo, os tratamentos realizados com duração de 1 ano ou mais, de acordo com Crits-Christoph \& Barber ${ }^{41}$, e de curta duração aqueles que foram realizados com um mínimo de 18 sessões ou por um período máximo de 11 meses. 


\section{Instrumentos}

\section{Questionário de efetividade}

O questionário de efetividade (EQ) é uma versão reduzida do questionário desenvolvido no The Consumer Report Study ${ }^{42}$. A tradução e adaptação do EQ para o português foi realizada por Freedman et al. ${ }^{22}$ O EQ é composto por 28 questões que investigam o motivo da busca de tratamento, estado emocional e físico, no início do tratamento e no momento atual, e percepção do paciente quanto à satisfação e aos benefícios com o tratamento psicoterápico.

Foi avaliado apenas o escore de efetividade (ES), que é obtido em três partes, cada uma derivada de questões separadas do EQ: progresso específico (100 pontos), satisfação do paciente com o tratamento de seus problemas pelo terapeuta (100 pontos) e progresso global (100 pontos). A pontuação 300 significa o máximo de efetividade terapêutica; 150 , nenhuma efetividade; e valores abaixo de 150 , efetividade negativa, no sentido de aumento de queixas psíquicas no curso do tratamento.

\section{Entrevista semi-estruturada}

A entrevista semi-estruturada foi audiogravada e, posteriormente, transcrita. Constituiu-se por uma questão abrangente no intuito de estimular verbalizações quanto ao estado atual do paciente. Quando necessário, foram realizadas intervenções de esclarecimento.

\section{Escala de avaliação global do funcionamento}

A escala de avaliação global do funcionamento (GAF) é descrita no eixo $\mathrm{V}$ do Manual Diagnóstico e Estatístico de Transtornos Mentais ${ }^{43}$. É uma escala bastante utilizada para rastrear o progresso clínico dos pacientes em termos globais, através da atribuição de uma medida única, que pode variar de 0 a 100 . A GAF foi aplicada às entrevistas de início de tratamento (encontradas dialogadas no arquivo da instituição) e às entrevistas de seguimento (final). Cada entrevista foi avaliada por, no mínimo, dois experts, havendo o cuidado para que os experts não aplicassem a GAF, a entrevista inicial e de seguimento do mesmo paciente. Foi realizado o cegamento para avaliação da GAF relativo à duração de tratamento dos pacientes, mas o mesmo procedimento não foi possível em relação à entrevista ser inicial ou de seguimento.

\section{Procedimentos}

Os pacientes foram contatados através de correspondência, seguida por um telefonema. No contato telefônico, foram esclarecidas as primeiras dúvidas do paciente sobre a pesquisa e marcada uma entrevista. Nessa entrevista, o paciente foi informado sobre o procedimento de pesquisa, foram esclarecidas dúvidas e preenchidos o consentimento livre e esclarecido e o EQ. Foi realizada, também nesse contato, a entrevista semi-estruturada. Essas etapas foram realizadas pela primeira autora.

A primeira entrevista de tratamento foi encontrada, relatada pelo psicoterapeuta do paciente, nos arquivos da instituição.

Após a digitação da primeira entrevista do paciente e da transcrição da entrevista de seguimento, experts independentes aplicaram a GAF em ambas as entrevistas.

\section{Instituição}

Os pacientes investigados são indivíduos que realizaram PP no Serviço de Atendimento dos Estudos Integrados de Psicoterapia Psicanalítica (ESIPP). O ESIPP é uma instituição de ensino, localizada na cidade de Porto Alegre, reconhecida pelo Conselho Federal de Psicologia e pela Associação Brasileira de Ensino em Psicologia, cuja principal finalidade é a formação de especialistas em PP.

\section{Tratamento}

A psicoterapia oferecida no serviço de atendimento do ESIPP é dirigida ao insight, realizada face a face, na freqüência de uma a três vezes por semana e sem utilização de manual. Ao longo do tratamento psicoterápico, os psicoterapeutas se reúnem semanalmente com supervisores da instituição (profissionais experientes em PP) para supervisão coletiva e individual dos casos atendidos. Realizam também, a cada semestre, um a três seminários de teoria e técnica psicanalítica na freqüência de uma vez por semana.

\section{Análise estatística}

O cálculo do tamanho amostral (17 pacientes em cada grupo) foi realizado através da comparação de médias (programa WINPEPI, módulo Compare, versão 1.45), para detectar uma diferença clinicamente relevante (tamanho de efeito $=1$ ou mais, isto é, um ou mais desvios padrão $=\mathrm{DP}$ ) entre as médias de alterações das GAF (inicial e seguimento) nos dois grupos de tratamento, considerando á $=0,05$ e poder de $80 \%$.

Para comparar as características dos grupos de tratamento entre si e não-participantes, utilizou-se: quiquadrado ou teste exato de Fisher para variáveis qualitativas, teste $t$ de Student para variáveis quantitativas com distribuição simétrica e teste de Mann-Whitney para variáveis quantitativas com distribuição assimétrica.

O teste $t$ de Student para amostras pareadas foi calculado para verificar a diferença entre os escores atribuídos às GAF (iniciais e de seguimento) pelos 
experts, considerando os dois grupos de tratamento. $\mathrm{Na}$ tentativa de chegar a uma pontuação mais homogênea, na medida em que o resultado do teste não encontrou diferença significativa entre as pontuações das GAF efetuadas pelos dois experts, um terceiro avaliador foi introduzido para pontuar a GAF, quando a diferença entre as duas pontuações anteriores era maior que mais ou menos um DP. Isso ocorreu em cinco casos de entrevista inicial e em sete casos de entrevista de seguimento no grupo 1; e em quatro casos de entrevista inicial e em três de entrevista de seguimento no grupo 2. O escore final foi realizado através da média entre as pontuações dos três avaliadores.

A análise de variância para medidas repetidas com dois fatores - ANOVA - foi utilizada para realizar a comparação entre os escores das GAF iniciais e de seguimento, ou seja, início e pós-tratamento, conforme o grupo investigado. A magnitude da diferença média entre os dois grupos foi verificada através do tamanho do efeito calculado pela metodologia de Cohen ${ }^{44}$. Para verificar a diferença das médias no ES entre os grupos, realizou-se o teste $t$ de Student para amostras independentes.

A correlação de Pearson foi calculada entre a GAF final e o ES (escores parciais e total). A correlação de Sperman foi utilizada para correlacionar tempo de experiência do terapeuta e resultado medido pela GAF final.

Os resultados foram analisados no programa Statistical Package for the Social Sciences (SPSS, versão 14.0), com nível de significância de 0,05 .

\section{Resultados}

\section{Características da amostra}

Foram encontrados, no arquivo da instituição, 82 pacientes que preencheram os critérios de inclusão na investigação. Destes, 23 não foram encontrados, nove não aceitaram participar da pesquisa, nove foram excluídos por estarem em algum tipo de tratamento na época do contato e sete desistiram da participação antes da entrevista.

A amostra ficou constituída por 34 pacientes (número mínimo encontrado no cálculo amostral), divididos em dois grupos: grupo $1-17$ pacientes que realizaram tratamento em um período de até 11 meses; e grupo $2-17$ pacientes com 1 ano ou mais de tratamento. A opção por esse ponto de corte foi uma tentativa de comparar tratamentos considerados de curta duração com tratamentos de longa duração.

Os pacientes foram contatados, em média, 20,9 meses $(\mathrm{DP}=10,7)$ e 29,9 meses $(\mathrm{DP}=16,7)$ após o término do tratamento psicoterápico, nos grupos 1 e 2 respectivamente.
Na Tabela 1, são apresentadas as características sociodemográficas dos pacientes participantes da pesquisa nos dois grupos de tratamento.

A Figura 1, a seguir, mostra os motivos da busca da psicoterapia em percentual nos grupos de tratamento investigados. Cabe ressaltar que foi possível ao paciente escolher, no questionário (EQ), mais de um motivo da busca de tratamento, ou seja, houve múltipla escolha.

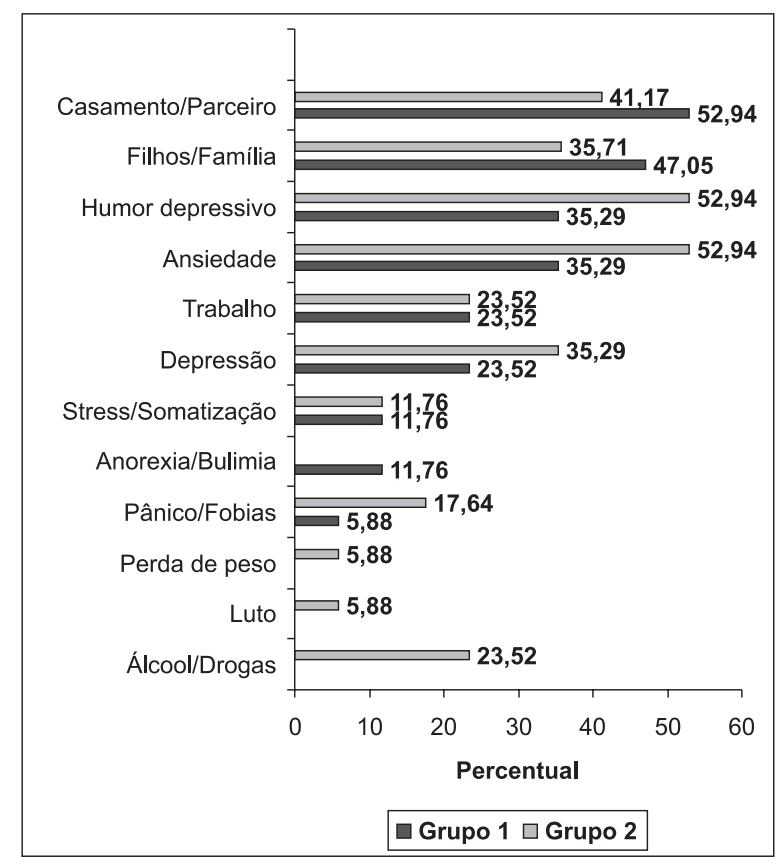

Figura 1 - Motivos da busca de tratamento

Para proceder a uma comparação, rastrearam-se as características sociodemográficas dos indivíduos nãoparticipantes da pesquisa, ou seja, 48 pacientes (23 que não foram encontrados, nove que recusaram a participação, nove excluídos por estarem em algum tipo de tratamento na época do contato e sete que desistiram), que serão apresentadas somente em comparação com os grupos dos participantes no item comparabilidade.

\section{Psicoterapeutas}

Os psicoterapeutas que conduziram os tratamentos são graduados em medicina ou psicologia e realizavam curso de especialização em psicoterapia na instituição. No grupo de pacientes tratados até 11 meses, a psicoterapia foi conduzida por 15 psicoterapeutas, sendo um médico e 14 psicólogos. Desses, 14 do sexo feminino e um do masculino, com a média de idade de 34,6 anos $(\mathrm{DP}=11,0)$ e tempo médio de experiência em 
Tabela 1 - Características sociodemográficas da amostra

\begin{tabular}{|c|c|c|}
\hline & $\begin{array}{c}\text { Grupo 1: pacientes até } 11 \text { meses } \\
\text { de tratamento } \\
(n=17)\end{array}$ & $\begin{array}{l}\text { Grupo 2: pacientes } 1 \text { ano ou } \\
\text { mais de tratamento } \\
(n=17)\end{array}$ \\
\hline Idade (anos), média (DP) & $32,4(9,5)$ & $34,1(8,2)$ \\
\hline \multicolumn{3}{|l|}{ Sexo } \\
\hline Feminino & $16(94,12 \%)$ & $13(76,47 \%)$ \\
\hline Masculino & $1(5,88 \%)$ & $4(23,53 \%)$ \\
\hline \multicolumn{3}{|l|}{ Estado civil } \\
\hline Casado & $6(35,29 \%)$ & $5(29,41 \%)$ \\
\hline Solteiro & $9(52,95 \%)$ & $7(41,18 \%)$ \\
\hline Divorciado/separado & $2(11,76 \%)$ & $5(29,41 \%)$ \\
\hline \multicolumn{3}{|l|}{ Instrução } \\
\hline Superior/superior incompleto & $11(64,71 \%)$ & $14(82,35 \%)$ \\
\hline Ensino médio/fundamental & $6(35,29 \%)$ & $3(17,65 \%)$ \\
\hline \multicolumn{3}{|l|}{ Residência } \\
\hline Porto Alegre & $16(94,12 \%)$ & $16(94,12 \%)$ \\
\hline Grande Porto Alegre & $1(5,88 \%)$ & $1(5,88 \%)$ \\
\hline Duração tratamento (meses), média (DP) & $6,4(2,6)$ & $24,7(9,3)$ \\
\hline Número de sessões, média (DP) & $31,1(13,4)$ & $148(77,9)$ \\
\hline \multicolumn{3}{|l|}{ Freqüência do tratamento } \\
\hline 1 vez por semana & $14(82,36 \%)$ & $11(64,70 \%)$ \\
\hline 2 vezes por semana & $2(11,76 \%)$ & $6(35,30 \%)$ \\
\hline 3 vezes por semana & $1(5,88 \%)$ & - \\
\hline \multicolumn{3}{|l|}{ Motivo do término } \\
\hline Alta & $1(5,88 \%)$ & $4236,53 \%$ \\
\hline Interrupção & $16(94,12 \%)$ & $13(76,47 \%)$ \\
\hline \multicolumn{3}{|l|}{ Uso de medicação } \\
\hline Sim & $1(5,88 \%)$ & $1(5,88 \%)$ \\
\hline Não & $16(94,12 \%)$ & $16(94,12 \%)$ \\
\hline \multicolumn{3}{|l|}{ Tratamento anterior } \\
\hline Sim & $11(64,70 \%)$ & $12(70,59 \%)$ \\
\hline Não & $6(35,30 \%)$ & $5(29,41 \%)$ \\
\hline \multicolumn{3}{|l|}{ Hospitalização psiquiátrica } \\
\hline Sim & $1(5,88 \%)$ & $2(11,76 \%)$ \\
\hline Não & $16(94,12 \%)$ & $15(88,24 \%)$ \\
\hline
\end{tabular}

$\mathrm{DP}=$ desvio padrão.

psicoterapia de 2,6 anos ( $D P=2,1)$. Dois psicoterapeutas conduziram dois tratamentos cada um neste grupo de pacientes.

O grupo de pacientes que realizou 1 ano ou mais de tratamento foi atendido por 13 psicoterapeutas, sendo um médico e 12 psicólogos. Desses, 12 do sexo feminino e um do masculino, com a média de idade de 39,3 anos (DP $=8,7)$ e tempo médio de experiência de 4,6 anos $(\mathrm{DP}=3,4)$. Nesse grupo, quatro psicoterapeutas realizaram dois tratamentos cada um com os pacientes da amostra. Cabe ressaltar, como em outras pesquisas ${ }^{19}$, neste estudo, dois psicoterapeutas realizaram tratamentos em ambos os grupos da amostra.

\section{Avaliadores}

Os 13 avaliadores independentes que participaram da pesquisa (dois do sexo masculino e 11 do feminino) possuem graduação em psicologia ou medicina, sendo seis psicólogos e sete psiquiatras. Destes, dois são psicanalistas e 11 têm pós-graduação em nível de mestrado (6) ou doutorado (5). A média das idades dos 
avaliadores era 41,2 anos ( $\mathrm{DP}=9,9)$, e a média do tempo de experiência em PP, 15,4 anos (DP = 9,2).

\section{Comparabilidade entre os grupos}

A comparação entre os grupos de tratamento é essencial para a análise posterior da efetividade da psicoterapia realizada. Não foram encontradas diferenças significativas entre os pacientes dos grupos 1 e 2 quanto a sexo, cidade de residência, idade, escolaridade, estado civil, freqüência de tratamento, uso de medicação, hospitalização psiquiátrica, tratamento anterior, sexo e idade do terapeuta, tempo de contato após o término e funcionamento global no início do tratamento. $\mathrm{O}$ tempo de experiência dos terapeutas que trataram o grupo 2, apesar de ser maior (2 anos aproximadamente), não foi estatisticamente significativo (teste Mann-Whitney $=92,000, p=0,066$ ). Houve diferença significativa entre os grupos (teste quiquadrado $=7,404, p=0,007)$ em relação ao motivo do término de tratamento: no grupo 2, ocorreram mais altas; no grupo 1, mais interrupções.

Comparando os pacientes dos grupos 1 e 2 quanto aos motivos da busca de tratamento, verifica-se diferença significativa apenas no referente à depressão (depressão, humor depressivo e luto), que foi maior no grupo 2 (teste exato de Fisher, $\mathrm{p}=0,039$ ).

Em relação à comparação dos não-participantes com até 11 meses de tratamento e dos pacientes do grupo 1 , não houve diferença significativa nas características comparadas - idade, estado civil, número de sessões, duração de tratamento, motivo da alta, cidade de moradia, escolaridade, idade, sexo e tempo de experiência do psicoterapeuta, exceto nas variáveis sexo do paciente os homens estão em maior número nos não-participantes (teste qui-quadrado $=5,929, \mathrm{p}=0,015$ ) - e tempo de contato após o término, que foi maior nos nãoparticipantes (teste $t$ de Student para amostras independentes $=5,987, \mathrm{p}<0,001$ ). O número maior de homens concentrou-se nos não-participantes que não foram encontrados.

Os não-participantes com 1 ano ou mais de tratamento, quando comparados com pacientes do grupo 2 , realizaram um número menor de sessões (teste $t$ de Student para amostras independentes $=-2,093, \mathrm{p}=$ $0,046)$, foram contatados em um tempo maior após a alta (teste $t$ de Student para amostras independentes = $5,044, p<0,001)$ e eram em sua maioria solteiros (teste qui-quadrado $=7,243, p=0,027$ ). Essas diferenças são significativas estatisticamente. A maioria de solteiros pertence aos não-participantes que não foram encontrados para fazer parte da investigação. Nas outras variáveis, idade, sexo, duração de tratamento, motivo da alta, cidade de moradia, escolaridade, idade, sexo e tempo de experiência do psicoterapeuta, não foram encontradas diferenças significativas.

\section{Efeito do tratamento}

Na Tabela 2, é apresentado o efeito de tratamento a partir da avaliação da GAF inicial e final (seguimento) nos dois grupos de tratamento.

Nesta investigação, não foi possível um grupocontrole; entretanto, foram comparados os dados encontrados com o índice de mudança média esperada (tamanho do efeito) em grupo-controle, elaborado por Leichsenring \& Rabung ${ }^{45}$, a partir de 26 estudos controlados randomizados de psicanálise e psicoterapia psicodinâmica (média $=0,12, \mathrm{DP}=0,19$ ). Esse tipo de comparação também foi utilizado por Leichsenring et al. ${ }^{13}$, em 2005, na divulgação dos resultados preliminares do chamado Göttingen study of effectiveness of

Tabela 2 - Efeito de tratamento

\begin{tabular}{lcccccc}
\hline & $\begin{array}{c}\text { GAF inicial } \\
\text { média (DP) }\end{array}$ & $\begin{array}{c}\text { GAF final* } \\
\text { média (DP) }\end{array}$ & P1 & P2 & P3 & $\begin{array}{c}\text { Tamanho do } \\
\text { efeito }\end{array}$ \\
\hline Grupo $1^{\dagger}(\mathrm{n}=17)$ & $57,18(8,38)$ & $72,38(12,12)$ & & & & 1,33 \\
Grupo $2^{*}(\mathrm{n}=17)$ & $57,04(5,78)$ & $74,62(10,18)$ & & & & 1,18 \\
Total $(\mathrm{n}=34)$ & $57,11(7,09)$ & $73,50(11,08)$ & $<0,001$ & 0,581 & 0,666 & 1,47 \\
\hline
\end{tabular}

GAF = escala de avaliação global do funcionamento; DP = desvio padrão.

Anova para medidas repetidas: $\mathrm{P} 1=\mathrm{p}$ associado à comparação GAF inicial versus final independente do grupo de tratamento; $\mathrm{P} 2=\mathrm{p}$ associado à comparação das variações da GAF inicial versus final conforme os dois grupos (interação); P3 = p associado à comparação entre os grupos, independente de GAF inicial/final.

\footnotetext{
*Seguimento.

† Pacientes com até 11 meses de tratamento.

‡ Pacientes com 1 ano ou mais de tratamento.
} 
psychoanalytic and psychodynamic therapy, isto é, os dados dos pacientes tratados em psicanálise. O tamanho do efeito encontrado no presente estudo, ao ser comparado com o índice de mudança - tamanho do efeito de grupo-controle ${ }^{45}$ - foi de 8,38 no total da amostra, 6,08 no grupo 1 e 8,01 no grupo 2, ou seja, uma diferença significativa tanto no total como nos grupos separadamente $(\mathrm{p}<0,001)$.

\section{Dados do questionário de efetividade}

A média do ES encontrado no EQ foi de 179,71 $(\mathrm{DP}=40,06)$ na amostra total; $180,30(\mathrm{DP}=40,87)$ no grupo 1; e 179,12 (DP = 40,48) no grupo 2. Não houve diferença significativa entre a pontuação do ES nos dois grupos de tratamento (teste $t$ de Student para amostras independentes $=0,084, p=0,933$ ).

A primeira pontuação do ES, explicitada na Tabela 3 , mediu o quanto o psicoterapeuta ajudou o paciente nos problemas específicos que o levaram ao tratamento.

Na segunda pontuação do ES, a medida avaliada foi a satisfação do paciente com o tratamento de seus problemas pelo terapeuta (Tabela 4).

A terceira pontuação do ES comparou o estado emocional do paciente no início do processo psicoterápico com o estado atual na entrevista de seguimento, isto é, o progresso global do paciente. Os pacientes marcaram uma das pontuações da seguinte escala: 1 - muito ruim, 2 algo ruim, 3 - mais ou menos, 4 - bastante bom e 5 muito bom. Dos 17 pacientes do grupo 1 que caracterizaram seu estado emocional como muito ruim, algo ruim ou mais ou menos no início da psicoterapia, $70,59 \%$ (12) avançaram uma até três pontuações na escala no final do tratamento, $23,53 \%$ (4) permaneceram no mesmo estado, e 5,88\% (1) piorou. No grupo 2, dos 17 pacientes que iniciaram a psicoterapia sentindo-se muito ruim, algo ruim ou mais ou menos, $64,71 \%$ (11) caracterizaram seu estado, no final do tratamento, melhor do que no início da psicoterapia, avançando uma a três pontuações na escala, $29,41 \%$ (5) permaneceram no mesmo estado, e $5,88 \%$ (1) piorou.

\section{Correlações}

A correlação entre a pontuação da GAF final (opinião dos experts) e o ES no EQ (opinião dos pacientes), na amostra total dos pacientes, foi de $r=$ $0,129$ ( $p=0,466)$, ou seja, uma correlação fraca e nãosignificativa. A correlação entre a GAF final e o estado emocional atual do paciente avaliado por ele mesmo foi de $\mathrm{r}=0,333(\mathrm{p}=0,054)$, isto é, uma correlação regular e no limite das significações.

Tabela 3 - Progresso específico de tratamento

\begin{tabular}{lcc}
\hline & $\begin{array}{c}\text { Grupo 1: até 11 meses } \\
\text { de tratamento } \\
(\mathbf{n}=\mathbf{1 7})\end{array}$ & $\begin{array}{c}\text { Grupo 2: 1 ano ou mais } \\
\text { de tratamento } \\
(\mathbf{n}=\mathbf{1 7})\end{array}$ \\
\hline Muito melhor & $52,94 \%(9)$ & $52,94 \%(9)$ \\
Melhor & $29,41 \%(5)$ & $29,41 \%(5)$ \\
Não fez diferença & $11,77 \%(2)$ & $17,65 \%(3)$ \\
Algo pior & $5,88 \%(1)$ & \\
Muito pior & & \\
Não tem certeza & & \\
\hline
\end{tabular}

Tabela 4 - Satisfação com o tratamento de seus problemas pelo psicoterapeuta

\begin{tabular}{lcc}
\hline & $\begin{array}{c}\text { Grupo 1: até 11 meses } \\
\text { de tratamento } \\
(\mathbf{n}=\mathbf{1 7})\end{array}$ & $\begin{array}{c}\text { Grupo 2: 1 ano ou mais } \\
\text { de tratamento } \\
(\mathbf{n}=\mathbf{1 7})\end{array}$ \\
\hline Completamente satisfeito & $17,65 \%(3)$ & $23,53 \%(4)$ \\
Muito satisfeito & $52,94 \%(9)$ & $29,41 \%(5)$ \\
Razoavelmente satisfeito & $17,65 \%(3)$ & $41,18 \%(7)$ \\
Algo insatisfeito & $11,76 \%(2)$ & \\
Muito insatisfeito & & $5,88 \%(1)$ \\
Completamente insatisfeito & & \\
\hline
\end{tabular}


No grupo 1, a correlação entre a GAF final e o EQ foi de $r=0,342(p=0,179)$, ou seja, representa uma correlação não-significativa. A correlação entre a GAF final e a pontuação no $\mathrm{EQ}$, que mediu a satisfação do paciente, foi de $r=0,0358(\mathrm{p}=0,159)$, significando uma correlação regular, mas sem poder estatístico.

A correlação, encontrada no grupo 2, entre a GAF final e o EQ não foi significativa $(\mathrm{r}=-0,121, \mathrm{p}=0,645)$. A correlação de Pearson entre a pontuação da GAF final e o estado emocional atual do paciente foi de $\mathrm{r}=$ $0,413(\mathrm{p}=0,099)$ : uma correlação regular $\mathrm{e}$ estatisticamente não-significativa.

Procedeu-se ao cálculo da correlação de Sperman entre o tempo de experiência dos psicoterapeutas e o resultado de tratamento obtido pela GAF final, sem encontrar significância $(r=0,007, p=0,969)$.

\section{Discussão}

Neste estudo naturalístico retrospectivo, avaliouse a efetividade da PP com pacientes adultos em dois grupos de tratamentos: grupo 1, com até 11 meses de psicoterapia, e grupo 2, com 1 ano ou mais de tratamento psicoterápico.

Ao ser realizada a comparabilidade entre os dois grupos de tratamento em termos demográficos, não foram encontradas diferenças significativas, exceto quanto ao motivo da busca de tratamento (informado pelo paciente no EQ) por depressão (depressão, humor depressivo e luto) e ao motivo do término de tratamento.

Um número significativamente maior de pacientes do grupo 2 buscou tratamento em função da depressão (depressão, humor depressivo e luto); entretanto, o grupo 1 apresentou número importante de pacientes com essa mesma queixa. O estudo comprova dados de outros informes, ou seja, os indivíduos que buscam a psicoterapia são, em sua maioria, os que apresentam sintomas de alterações de humor ${ }^{46-48}$, assim como as áreas em que os indivíduos mais mostram sofrimento quando procuram psicoterapia são: casamento/parceiro, família/filhos e conflitivas com o trabalho ${ }^{46,47,49}$.

No grupo 2, ocorreram mais altas, e no grupo 1, mais interrupções: uma diferença compreensível, uma vez que o grupo 2 é composto pelos pacientes que se trataram por mais tempo - o que proporciona uma maior probabilidade de alta - e pelo fato de que as interrupções tendem a acontecer mais no início dos tratamentos.

$\mathrm{Na}$ literatura especializada, a variedade de definições do que é considerado abandono e interrupção é fator confundidor ${ }^{50-52}$. Independentemente dessas definições, o percentual de pacientes que desistem da psicoterapia (abandono propriamente dito ou interrupção comunicada), em diferentes idades, grupos diagnósticos e modalidades de tratamento, variam de
30 a $60 \%{ }^{53}$. Em estudos realizados em serviços de atendimento psicoterápico no Brasil, as taxas de desistência variam entre 35 e $68,7 \%$ da população atendida $^{54-56}$. Esse alto índice de interrupções chama a atenção, mas os dados da amostra pesquisada mostram taxas ainda maiores: no grupo 1, houve 16 interrupções (94,12\%), e no grupo 2,13 interrupções $(76,47 \%)$.

$\mathrm{O}$ processo de término psicoterápico envolve a vivência de emoções importantes, que podem levar a uma maior ou menor dificuldade na condução do tratamento ao seu final. Paciente e psicoterapeuta terão que se deparar com sentimentos ligados à separação, à elaboração de lutos e à aceitação dos limites tanto do paciente quanto da dupla psicoterápica. Portanto, as variações em torno do processo do término de tratamento são muito complexas, e o término, muitas vezes, caracteriza-se por interrupções com pacientes retornando mais tarde ao tratamento ${ }^{57}$. Sabe-se que menos de $20 \%$ dos pacientes, em centros de saúde mental comunitários, realizam tratamento cujo término é de mútuo acordo ${ }^{50}$. Nossa amostra confirma essas afirmações: além de ter sido constituída por uma maioria de pacientes que interromperam os tratamentos, um número significativo havia recebido algum tipo de tratamento anterior.

Outro ponto a discutir em relação ao número elevado de interrupções é o fato de os psicoterapeutas serem iniciantes. A inexperiência dos psicoterapeutas pode levar à idealização e à expectativa altamente perfeccionista, aspirando a uma mudança estrutural ou a uma resolução transferencial que não seja possível com a realidade dos pacientes; pode também favorecer erros contratransferenciais, e o psicoterapeuta deixa de apoiar o término em função de suas ambições pessoais, na maioria das vezes, bem maiores que a do próprio paciente $^{57}$. Isso significa que a interpretação do psicoterapeuta do término da psicoterapia como interrupção de tratamento pode, muitas vezes, estar influenciada por esses fatores, levando à distorção do que realmente ocorreu e superestimando as interrupções.

Conforme estudo de Sandell et al. ${ }^{10}$, os psicoterapeutas mais antigos tendem a alcançar melhores resultados com seus casos, obtendo uma correlação positiva entre a quantidade de tempo que uma pessoa vem trabalhando e o resultado de seus tratamentos. Não foi encontrada correlação significativa entre o tempo de experiência do psicoterapeuta e o resultado de tratamento nesta amostra. Acredita-se que, tratando-se de psicoterapeutas com pouca experiência em psicoterapia, a diferença de tempo de experiência entre eles é muito pequena e, possivelmente, não chegue a possuir uma significância no desempenho clínico.

A amostra constituída, predominantemente, por pacientes do sexo feminino ratifica dados de outros estudos em relação à prevalência de pacientes mulheres 
na busca por psicoterapia ${ }^{12,16,19,58,59}$. O sexo dos psicoterapeutas, também em sua grande maioria feminino, mostra a tendência de um número maior de psicoterapeutas mulheres do que homens entre os profissionais em PP no Brasil. A presença de psicoterapeuta do sexo masculino, que poderia ser interpretado como um fator de confusão, ficou controlada, já que ocorreu em igual número em ambos os grupos de tratamento (um em cada grupo).

É pertinente assinalar que as diferenças nas pontuações das GAF iniciais dos pacientes, nos grupos de comparação, não foram significativas, ou seja, os pacientes iniciaram seus tratamentos com um nível de funcionamento global semelhante. Esse dado é relevante, considerando que o diagnóstico inicial dos pacientes não foi realizado e que há apenas uma idéia da constelação clínica da amostra através do rastreamento dos motivos da busca de tratamento.

Em relação à comparação dos participantes e nãoparticipantes da pesquisa, as diferenças significativas encontradas tendem a justificar, em parte, a nãoparticipação dos pacientes, como: número de sessões menor e contato realizado após tempo maior da terminação. O percentual maior de não-participantes até 11 meses de tratamento do sexo masculino foi significativo quando comparado ao grupo 1, bem como o número de solteiros dentre os que não participaram com 1 ano ou mais de tratamento, quando comparados ao grupo 2. Esse dado é interessante, pois ocorreu, em sua maioria, nos não-participantes que não foram encontrados. É possível levantar a hipótese de que ser do sexo masculino e solteiro pode levar o indivíduo à busca mais intensa de novas oportunidades, o que justificaria parcialmente o fato de não terem sido encontrados.

Em relação à representatividade da amostra, um questionamento sempre possível é se aqueles pacientes que foram encontrados e participaram da pesquisa são os que mais atingiram seus objetivos de tratamento, sendo os não-encontrados ou os que negaram a participação aqueles para os quais o tratamento falhou.

Caso sejam considerados, como número total, os pacientes encontrados no arquivo da instituição, ou seja, 82 pacientes, o índice de perdas seria grande $(58,54 \%)$, porém no mesmo percentual da pesquisa de resultados conduzida por Freedman et al. ${ }^{58}$. Retirando desse cálculo de perdas os pacientes não-contatados (23) e os excluídos por estarem realizando outro tratamento (9), o índice passa a 32\% (nove que não aceitaram participar e sete que desistiram no andamento da pesquisa) da amostra de 50 pacientes. Esse índice está acima das perdas encontradas nos estudos de Leuzinger-Bohleber et al. ${ }^{12}$ e Grande et al. ${ }^{19}$; no entanto, abaixo das perdas apresentadas em outros estudos de resultado ${ }^{10,15,16}$.
A diferença estatisticamente significativa $(\mathrm{p}<$ 0,001 ) encontrada no total da amostra (GAF inicial versus final) demonstra que o nível de funcionamento global dos pacientes melhorou, independentemente do grupo de tratamento investigado. Porém, não foram encontradas evidências de interação entre duração de tratamento e resultado $(\mathrm{p}=0,581)$, ou seja, com esta amostra, a duração do tratamento por si só não exerceu influência na melhora do funcionamento global, pois os pacientes, em ambos os grupos, melhoraram na mesma proporção. Esse dado difere de investigações anteriores, cujos resultados comprovam que a duração ou tempo de tratamento possuem relação direta com o resultado ${ }^{10,22,58,60,61}$. A ampliação do número de participantes desse estudo é necessária, pois com uma amostra maior talvez fosse possível detectar diferenças no resultado do tratamento dos grupos investigados.

Desse modo, algumas questões são possíveis de serem feitas: o paciente poderia apresentar uma melhora intensa no funcionamento global no início do tratamento? A GAF seria um instrumento sensível o bastante para detectar mudanças estruturais e transferenciais que geralmente acontecem em tratamentos de longa duração? A melhora sintomática no início dos tratamentos e a possível falta de sensibilidade da GAF para mudanças mais profundas poderiam justificar, de certa forma, os resultados semelhantes nos dois grupos, ou seja, seria uma questão para futuras pesquisas.

Outro fator a considerar, apesar de controverso, é o que Malan ${ }^{62}$ denominou de "eros terapêutico", ou seja, o entusiasmo que toma conta dos psicoterapeutas iniciantes. O autor descreveu uma disposição contagiante dos psicoterapeutas que leva a repercussões positivas sobre o resultado da terapia, apesar de mais tarde ter proposto o abandono dessa hipótese ${ }^{63}$. Entretanto, esta parece ser uma constatação clínica à espera de uma melhor compreensão ou comprovação em pesquisa.

De maneira geral, é esperado que psicoterapeutas com maior experiência possibilitem melhores resultados de tratamento, como comprovado por Sandell et al. ${ }^{10}$ Portanto, possivelmente com psicoterapeutas mais experientes, ter-se-ia uma probabilidade maior de encontrar uma diferença significativa entre o resultado de tratamento dos pacientes dos grupos 1 e 2 .

O relevante a considerar é que, com esta amostra, fica comprovado que, analisada individualmente, a duração de tratamento, necessariamente, não é fator decisivo para o resultado psicoterápico; certamente, outros fatores devem ser considerados, como a relação terapêutica, a qualidade das relações objetais, o potencial auto-reflexivo e a reação às interpretações.

O tamanho do efeito de tratamento encontrado (GAF inicial versus final) pode ser considerado grande 
$\left(C o h e n^{44}\right)$ na amostra total e nos grupos separadamente. Esses índices, quando comparados ao índice de mudança média (tamanho do efeito), elaborado por Leichsenring \& Rabung ${ }^{45}$, foram significativamente maiores, independentemente do grupo de tratamento (índices semelhantes aos encontrados por Leichsenring et al. ${ }^{13}$ ). Esses dados, além de ratificarem os resultados encontrados, permitem considerar que a melhora encontrada nos pacientes tem uma probabilidade maior de ser atribuída ao tratamento psicoterápico do que a qualquer outra variável.

O número elevado de interrupções, apontado anteriormente, contrasta com a opinião dos pacientes sobre o tratamento vivenciado, emitida através do preenchimento do EQ. Em sua maioria, os pacientes de ambos os grupos sentiram-se completamente satisfeitos, muito satisfeitos ou razoavelmente satisfeitos com o tratamento, dado pelo terapeuta, aos seus problemas; consideraram que a psicoterapia/psicoterapeuta tornou as coisas muito melhores ou melhores em relação ao motivo da busca de tratamento e caracterizaram seu estado, no final do tratamento, como melhor do que no início da psicoterapia.

Especificamente em relação à satisfação de tratamento, somando o percentual dos pacientes completamente satisfeitos, muito satisfeitos e razoavelmente satisfeitos, o grupo 1 apresentou índice de $88,24 \%$ de satisfação ( $11,76 \%$ de insatisfação), e o grupo $2,94,12 \%$ de satisfação $(5,88 \%$ de insatisfação). Esses dados reforçam a idéia de que a maioria dos pacientes fica satisfeita com resultados em um tempo menor do que aquele que seus psicoterapeutas considerariam ideal e terminam o tratamento, muitas vezes, à revelia do psicoterapeuta, que ambiciona mais melhoras ao seu paciente; ou seja, para o paciente houve alta, para o psicoterapeuta, interrupção. Isso seria o mesmo que dizer que o número de altas está subestimado pelos psicoterapeutas. Esses achados podem ser referendados pelo estudo de Leuzinger-Bohleber et al. ${ }^{12}$, no qual os analistas se mostraram muito mais críticos em relação aos resultados do tratamento que os pacientes.

O escore médio de efetividade do EQ de 179,71 ( $\mathrm{DP}=40,056)$, na amostra total, é relativamente inferior ao encontrado em outras pesquisas ${ }^{60,64}$. Deve-se levar em conta que as amostras, nestes estudos, não são homogêneas, e que tais diferenças podem expressar os contextos em que as investigações foram realizadas. Quanto à comparação do EQ nos grupos de tratamento, não houve diferença significativa na pontuação do ES, ou seja, os pacientes possuem opiniões semelhantes quanto ao tratamento, independentemente de pertencer a um ou a outro grupo de tratamento investigado.

Não foi encontrada correlação significativa entre a pontuação da GAF final e o ES no EQ na amostra total dos pacientes e nos grupos de tratamento. Isso significa que a opinião dos experts, emitida através da pontuação da GAF final, não apresenta correlação com a opinião dos pacientes, avaliada através do ES. Os dados mostram uma tendência de correlação (regular e no limite das significações) entre a GAF final e o estado emocional atual do paciente, avaliado por ele mesmo.

É possível supor que, apesar de ambos, experts e pacientes, considerarem, de maneira geral, que a psicoterapia oferecida foi efetiva, houve discrepância quanto à opinião específica de cada caso. Além disso, há que ser considerada a dimensão subjetiva de cada paciente em sua avaliação do próprio tratamento, o que leva a possíveis inclusões de suas fantasias, expectativas e transferências como aparente sinal de melhores ou piores resultados. Tratando-se de duas medidas diferentes (GAF e EQ), não foi possível verificar se houve maior rigor por parte dos avaliadores ou dos pacientes na avaliação dos tratamentos.

Cabe apontar as limitações do estudo: amostra pequena, coleta de dados retrospectiva, utilização de psicoterapeutas em formação, uso de poucos instrumentos de medidas, ausência de grupo-controle e de diagnóstico dos pacientes investigados.

Existe a possibilidade de, com uma amostra ampliada, ser possível detectar diferenças no resultado de tratamento dos pacientes do grupo 1 e do grupo 2, ou seja, encontrar uma interação entre duração e resultado de tratamento, como em outros estudos, considerando que, com o número de participantes dessa amostra, o poder estatístico do resultado encontrado é pequeno ( 8 a 15\%). Entretanto, com esse número de pacientes (17 em cada grupo), teria sido possível detectar uma diferença clinicamente relevante (um ou mais DP) entre as médias de alterações das GAF (iniciais e de seguimento) nos dois grupos de tratamento (poder de $80 \%$ e á $=0,05)$.

A reconstrução do passado, após a ocorrência do desfecho, pode introduzir um viés de memória na percepção do paciente em relação ao tratamento psicoterápico. Contudo, a avaliação de que o tratamento, de uma forma geral, foi efetivo, tanto na opinião dos pacientes como na opinião dos experts, sugere que o viés de memória não distorceu de forma significativa a apreciação dos resultados. A vantagem de um estudo retrospectivo está em não influenciar o processo e o resultado da psicoterapia, o que, neste meio, é algo significativo, em função de certa resistência à pesquisa por parte dos psicoterapeutas.

O tratamento conduzido por psicoterapeutas inexperientes poderia ser um fator confundidor, mas, como os resultados de melhora na mostra foram significativos, esse fato contribui para a comprovação da efetividade de tratamento, mesmo com profissionais iniciantes. O que pode ter ficado menos claro é a 
comparação realizada entre resultado e duração de tratamento, pois, como já mencionado, talvez com psicoterapeutas mais experientes fossem encontradas diferenças significativas no resultado entre os dois grupos de tratamento.

A utilização de múltiplas medidas parece ser o mais adequado para avaliar resultados em psicoterapia, ${ }^{65,66}$. Os instrumentos dessa investigação foram limitados: entrevista semi-estruturada, questionário auto-aplicável e escala clínica (GAF) aplicada por experts. A GAF e a entrevista são instrumentos bastante utilizados em pesquisas, porém o EQ tem limites, como a não-validação para o português (apesar de já ter sido utilizada no Brasil uma tradução adaptada por Freedman et al. ${ }^{22}$ ) e o fato de não estar bem consolidado como instrumento de pesquisa.

A condução das entrevistas de seguimento pela primeira autora deste artigo pode significar um viés do pesquisador, mas foi balanceada pela avaliação da entrevista por experts independentes. Não foi possível cegar as entrevistas avaliadas com relação ao seu momento de aplicação (entrevistas iniciais ou de seguimento), o que pode ter gerado certa influência no resultado das avaliações. Entretanto, houve o cuidado para que os experts não avaliassem a entrevista inicial e de seguimento do mesmo paciente.

A ausência de um grupo-controle de pesquisa decorre, principalmente, de dificuldades éticas e logísticas. Os resultados, entretanto, são fortalecidos pela comparação realizada com o índice de mudança média esperada em grupo-controle elaborado por Leichsenring \& Rabung ${ }^{45}$, na qual foi detectada uma mudança significativamente maior no grupo de tratamento psicoterápico.

A falta de um diagnóstico inicial da amostra é outra limitação. Neste estudo, houve certa compensação da ausência de diagnóstico, através do rastreamento dos motivos de busca de tratamento e do fato de o nível de funcionamento global dos pacientes não ter diferido no início do tratamento. É relevante mencionar que, segundo Jones ${ }^{67}$, não há uma relação direta entre 0 diagnóstico formal e o processo de mudança em psicoterapia, e raramente ele é útil na antecipação de eventos do tratamento.

\section{Conclusões}

Os resultados do presente estudo indicam que os pacientes tratados com PP, na amostra investigada, melhoraram seu funcionamento global e possuem uma opinião favorável sobre os ganhos com o tratamento psicoterápico. No entanto, a opinião dos experts e a dos pacientes não foram correlacionadas positivamente, apesar de ambas demonstrarem a melhora significativa dos pacientes. A duração de tratamento isolada não foi determinante para o resultado da PP. Esta é certamente uma área de grande relevância para futuras pesquisas em PP.

Investigar é um dos desafios dos profissionais da área de saúde mental, independentemente do alcance, limites e dificuldades em pesquisar sobre a efetividade dos tratamentos. Como dizem De La Parra et al. ${ }^{68}$, "investigar representa um imperativo ético: é realmente eficaz o que estamos propondo no momento que fazemos a indicação de psicoterapia?".

\section{Agradecimento}

Agradecemos ao Dr. Falk Leichsenring e ao Dr. Rabung pelo envio do artigo Change norms: a complementary approach to the issue of controls groups in psychotherapy outcome research (na época ainda nãopublicado) para a utilização nesta investigação.

\section{Referências}

1. Vitória G. A busca da felicidade. Rev Isto É. 1995;1356:100-7.

2. Cavalcante R. A psicanálise no divã. Rev Superinteressante. 2002;181:42-50

3. Lima JG. Freud é um vencedor: entrevista com Renato Mezan. Rev Veja. 2006;39(17):13-7.

4. Solms M. Freud está de volta. Sci Am Bras. 2004;3(25):51-6.

5. Hobson AL. Freud está de volta? Como num pesadelo. Sci Am Bras. 2004;3(25):57.

6. Eizirik CL. Psychoanalysis as a work in progress. Int J Psychoanal. 2006;87:645-50.

7. Kandel ER. A biologia e o futuro da psicanálise: um novo referencial intelectual para a psiquiatria revisitado. Rev Psiquiatr RS. 2003;25:139-65.

8. Nunes MLT, Lhullier AC. Histórico da pesquisa empírica em psicoterapia. Rev Bras. Psicoter. 2003;5(1):97-112.

9. Freud S. Estudos sobre histeria. Rio de Janeiro: Imago; 1989. Vol. VII. p. 12-115.

10. Sandell R, Blomberg J, Lazar A, Carlsson J, Broberg J, Schubert J. Varieties of long-term outcome among patients in psychoanalysis and long-term psychotherapy. A review of findings in the Stockholm Outcome of Psychoanalysis and Psychotherapy Project. Int J Psychoanal. 2000;81(Pt 5):921-42.

11. Brockmann J, Schluter T, Eckert J. [Therapy goals, change of goals and goal attainment in the process of psychoanalytically oriented and behavior long-term therapy - a comparative study from the private practices of insurance-registered psychotherapists]. Psychother Psychosom Med Psychol. 2003;53(3-4):163-70.

12. Leuzinger-Bohleber M, Stuhr U, Ruger B, Beutel M. How to study the quality of psychoanalytic treatments and their long-term effects on patients well-being: a representative, multi-perspective followup study. Int J Psychoanal. 2003;84(Pt 2):263-90.

13. Leichsenring F, Biskup J, Kreische R, Staats H. The Gottingen study of psychoanalytic therapy: first results. Int J Psychoanal. 2005;86(Pt 2):433-55

14. Huber D, Klug, G e Von Rad, M. Die Münchner Prozess: Outcome Studie. Ein Vergleich Zwischen Psychoanalysen und psychodynamischen Psichotherapien unter besonderer Berücksichtigung therapiespezifischer Ergebnisse. In: Stuhr U, Leuzinger-Bohleber M, Beutel M, eds. Psychoanalytische Langzeittherapien. Stuttgart: Kohlhmmer; 2001. 
15. Bond M, Perry JC. Long-term changes in defense styles with psychodynamic psychotherapy for depressive, anxiety, and personality disorders. Am J Psychiatry. 2004;161(9):1665-71.

16. Wilczek A, Barber JP, Gustavsson JP, Asberg M, Weinryb RM. Change after long-term psychoanalytic psychotherapy. J Am Psychoanal Assoc. 2004;52(4):1163-84.

17. Gerber AJ, Fonagy P, Target M, Bateman A, Higgit A. Structural and symptomatic change in psychoanalysis and psychodynamic psychotherapy of young adults: a qualitative study of treatment process and outcome, 2004. Disponível em: http://www.ipa.org.uk/ research/pdf/gerber.pdf. Acessado jul 2006.

18. Bond M, Perry JC. Psychotropic medication use, personality disorder and improvement in long-term dynamic psychotherapy. J Nerv Ment Dis. 2006;194(1):21-6.

19. Grande T, Dilg R, Jakobsen T, Keller W, Krawietz B, Langer M, et al. Differential effects of two forms of psychoanalytic therapy: results of the Heidelberg-Berlin study. Psychother Res. 2006;16(4):470-85.

20. Wallerstein RS. Outcome research. In: Cooper A, Person ES, eds. The American psychiatric publishing textbook of psychoanalysis. Washington (DC): Am Psychiatric; 2005. p. 301-15.

21. Bernardi RE. Investigación clínica e empírica sistemática em psicoanálisis. In: Lhullier AC, org. Novos modelos de investigação em psicoterapia. Pelotas: Educat; 1998. p. 23-42.

22. Freedman N, Hoffenberg J, Borus N, Eizirik C, Knijinik D. Tiempo en tratamiento: observaciones de Mid-Manhattan y Porto Alegre, Brasil. Invest Psicoan Psicoter. 2001:87-101.

23. Rascón SR, Corona PC, Latirgue T, Rios JM, Garza DL. A successful trial utilizing the Leuzinger-Bohleber methodology for evaluation of psychoanalytic treatment: preliminary report. Int $\mathrm{J}$ Psychoanal. 2005;86(Pt 5):1425-40.

24. Moreno CML, Schaleyeff C, Acosta S, Vernengo P, Roussos A, Lerner BD. Evaluation of psychic change through the application of empirical and clinical techniques for a 2-year treatment: a single case study. Psychother Res. 2005;15(3):199-209.

25. Gabbard, GO. Major modalities: psychoanalytic/psychodynamic. In: Gabbard GO, Beck JS, Holmes J. Oxford textbook of psychotherapy. EUA: Oxford University; 2005. cap 1. p. 3-13.

26. Howard KI, Kopta SM, Krause MS, Orlinsky DE. The dose-effect relationship in psychotherapy. Am Psychol. 1986;41(2):159-64.

27. Kopta SM, Howard KI, Lowry JL, Beutler LE. Patterns of symptomatic recovery in psychotherapy. J Consult Clin Psychol. 1994;62(5):1009-16.

28. Milrod B, Busch F, Leon AC, Shapiro T, Aronson A, Roiphe J, et al. Open trial of psychodynamic psychotherapy for panic disorder: a pilot study. Am J Psychiatry. 2000;157(11):1878-80.

29. Dare C, Eisler I, Russell G, Treasure J, Dodge L. Psychological therapies for adults with anorexia nervosa: randomized controlled trial of out-patient treatments. Br J Psychiatry. 2001;178:216-21.

30. Junkert-Tress B, Schnierda U, Hartkamp N, Schmitz N, Tress W. Effects of short-term dynamic psychotherapy for neurotic, somatoform, and personality disorders: a prospective 1-year followup study. Psychother Res. 2001;11(2):187-200.

31. Abbass A. Intensive Short-term Dynamic Psychotherapy in a private psychiatric office: clinical and cost effectiveness. Am J Psychother. 2002;56(2):225-32.

32. Burnand Y, Andreoli A, Kolatte E, Venturini A, Rosset N. Psychodynamic psychotherapy and clomipramine in the treatment of major depression. Psychiatr Serv. 2002;53(5):585-90.

33. Cooper PJ, Murray L, Wilson A, Romaniuk H. Controlled trial of the short- and long-term effect of psychological treatment of postpartum depression. I. Impact on maternal mood. Br J Psychiatry. $2003 ; 182: 412-9$

34. Hilsenroth MJ, Ackerman SJ, Blagys MD, Baity MR, Mooney MA. Short-term psychodynamic psychotherapy for depression: an examination of statistical, clinically significant, and techniquespecific change. J Nerv Ment Dis. 2003;191(6):349-57.

35. Svartberg M, Stiles TC, Seltzer MH. Randomized, controlled trial of the effectiveness of short-term dynamic psychotherapy and cognitive therapy for cluster C personality disorders. Am J Psychiatry. 2004;161(5):810-7.

36. Muran JC, Safran JD, Samstag LW, Winston A. Alliance-focused treatment for personality disorders. Psychother Theor Res Pract Train. 2005;42(4):532-45.

37. Maina G, Forner F, Bogetto F. Randomized controlled trial comparing brief dynamic and supportive therapy with waiting list condition in minor depressive disorders. Psychother Psychosom. 2005;74(1):43-50.

38. Hersoug AG, Bogwald KP, Hoglend P. Changes of Defensive Functioning: Does Interpretation Contribute to Change? Clin Psychol Psychother. 2005;12(4):288-96.

39. Stiles WB, Barkham M, Twigg E, Mellor-Clark J, Cooper M. Effectiveness of cognitive-behavioural, person-centred and psychodynamic therapies as practised in UK National Health Service settings. Psychol Med. 2006;36:(4):555-66.

40. Leichsenring F. Are psychodynamic and psychoanalytic therapies effective? A review of empirical data. Int J Psychoanal. 2005:86(Pt 3):841-68.

41. Crits-Christoph P, Barber JP. Long-term psychotherapy. In: Ingram RE, Snyder CR. Handbook of psychological change: psychotherapy processes an practices for the 21st century. New York: Wiley; 2000. p. $455-73$.

42. Seligman MEP. The effectiveness of psychotherapy. The Consumer Reports study. Am Psychol. 1995;50:965-74

43. American Psychiatric Association. Manual diagnóstico e estatístico de transtornos mentais. 4th ed. Porto Alegre: Artmed; 1995.

44. Cohen J. Statistical power analysis for the behavioral sciences. $2 \mathrm{dn}$ ed. Hilllsdale (NJ): Erlbaum; 1988.

45. Leichsenring F, Rabung S. Change norms: a complementary approach to the issue of controls groups in psychotherapy outcome research. Psychother Res. 2006;16:594-605.

46. Urtiaga ME, Almeida G, Vianna MED, Santos MV, Botelho S. Fatores preditivos de abandono em psicoterapias: um estudo na clínica Sérgio Abuchaim. J Bras Psiquiatr. 1997;46:279-83.

47. Pinheiro SD. Vínculo e abandono em psicoterapia psicanalítica [dissertação]. Porto Alegre: Pontifícia Universidade Católica do Rio Grande do Sul; 2002.

48. Kessler RC, Berglund P, Demler O, Jin R, Koretz D, Merikangas $\mathrm{KR}$, et al. The epidemiology of major depressive disorder: results from the National Comorbidity Survey Replication (NCS-R). JAMA. 2003;289(23):3095-105.

49. Montado G. Psicoterapia focal psicoanalítica: investigación de proceso y resultados. In: Bernardi R, Defey D, Hebert EJ, Fiorini H, Fonagy P, Gril S, et al. Psicoanalisis focos y aperturas. Montevideo: Agora/Psicolibros; 2001. p. 248-61.

50. Beck NC, Lamberti J, Gamache M, Lake EA, Fraps CL, McReynolds WT, et al. Situational factors and behavioral self-predictions in the identification of clients at high risk to drop out psychotherapy. J Clin Psychol. 1987;43(5):511-20.

51. Garfield SL. Research on client variables in psychotherapy: research pertaining to continuation in psychotherapy. In: Bergin A, Garfield S. Handbook of psychotherapy and behavior change. New York: Wiley; 1994. p. 190-228.

52. Lhullier AC. Abandono de tratamento em psicoterapias realizadas em uma clinica-escola. [tese]. Porto Alegre: Pontifícia Universidade Católica do Rio Grande do Sul; 2002.

53. Baekeland F, Lundwall L. Dropping out of treatment: a critical review. Psychol Bull. 1975;82(5):738-83.

54. Lopez MA. Considerações sobre o atendimento fornecido por clínicas-escola de psicologia. Arq Bras Psicol. 1983;35(2):123-35.

55. Carvalho RMLL, Térziz A. Caracterização da população atendida na clínica-escola do Instituto de Psicologia: PUCCAMP. Est Psicol (Campinas). 1988;5(1):112-25.

56. Lhullier AC, Nunes MLT, Antochevis AF, Porto AM, Figueiredo D. Mudança de terapeuta e abandono da psicoterapia em uma clínicaescola. Aletheia. 2000;11:7-11.

57. Gabbard GO. Elaboração e término. In: Gabbard GO. Psicoterapia psicodinâmica de longo prazo: texto básico. Porto Alegre: ArtMed; 2005. p. 161-78.

58. Friedman RC, Garrison WB, Bucci W, Gorman BS. Factors affecting change in private psychotherapy patients of senior psychoanalysts: an effectiveness study. J Am Acad Psychoanal Dyn Psychiatry. 2005;33(4):583-610.

59. Vargas F. Abandono de psicoterapia em instituição de formação de 
psicoterapeutas [dissertação]. Porto Alegre: Pontifícia Universidade Católica do Rio Grande do Sul; 2002.

60. Freedman N, Hoffenberg JD, Vorus N, Frosch A. The effectiveness of psychoanalytic psychotherapy: the role of treatment duration, frequency of sessions and the therapeutic relationship. J Am Psychoanal Assoc. 1999;47(3):741-72.

61. Kordy H, Von Rad M, Senf W. Empirical hypotheses on the Psychotherapeutic treatment of psychosomatic patients and short and long-term time unlimited psychotherapy. Psychother Psychosom. 1989;52(1-3):155-63.

62. Malan D. Toward the validation of dynamic psychotherapy. Nem York: Plenun Medical; 1976.

63. Malan D. Resultados sobre o segundo estudo em psicoterapia breve. In: Malan D. As fronteiras da psicoterapia breve: um exemplo de convergência entre pesquisa e prática Médica. Porto Alegre: Artmed; 1981. p. 57-65.
64. Hartman S, Zepf S. Saarland study of psychotherapy effectiveness and patient satisfaction. In: Fonagy P, ed. An open door review of outcome studies in psychoanalysis. London: International Psychoanalytical Association; 2002. p. 147-9.

65. Edmonstone Y, Freeman C. Research in psychotherapy. In: Freeman $\mathrm{C}$, Tyler P, eds. Research methods in psychiatry: a beginner's guide. EUA: American Psychiatric; 1992. p. 209-32.

66. Aveline M, Strauss B, Stiles WB. Psychotherapy research. In: Gabbard GO, Beck JS, Holmes. Oxford textbook of psychotherapy. EUA: Oxford; 2005. p. 449-62.

67. Jones EE. Therapeutic action: a guide to psychoanalytic psychotherapy. New Jersey: Aronson; 2000.

68. de la Parra G, von Bergen A, del Rio M. Primeros hallazgos de la aplicación un instrumento que mide resultados psicoterapéuticos en una muestra de pacientes y de población general. Rev Chil NeuroPsiquiatr. 2002;40(3):201-9. 